\title{
Miliary tuberculosis with no pulmonary involvement in myelodysplastic syndromes: a curable, yet rarely diagnosed, disease: case report and review of the literature
} Ioannis K Neonakis*1, Michael G Alexandrakis², Zoe Gitti1 ${ }^{1}$, George Tsirakis², Elias Krambovitis ${ }^{3}$ and Demetrios A Spandidos ${ }^{1}$

\author{
Address: ${ }^{1}$ Mycobacteriology Laboratory, Department of Clinical Bacteriology, Parasitology, Zoonoses and Geographical Medicine, University \\ Hospital of Heraklion, Voutes, 71201 Heraklion, Greece, ${ }^{2}$ Department of Hematology, University Hospital of Heraklion, Voutes, 71201 \\ Heraklion, Greece and ${ }^{3}$ Microbiology and Parasitology Laboratory, Department of Veterinary Medicine, School of Health Sciences, University of \\ Thessaly, 224 Trikalon Str., 43100 Karditsa, Greece \\ Email: Ioannis K Neonakis* - ineonakis@gmail.com; Michael G Alexandrakis - alexandm@med.uoc.gr; Zoe Gitti - zgitti@gmail.com; \\ George Tsirakis - geotsir@yahoo.com; Elias Krambovitis - krambo@imbb.forth.gr; Demetrios A Spandidos - spandidos@spandidos.gr \\ * Corresponding author
}

Published: I 3 March 2008

Annals of Clinical Microbiology and Antimicrobials 2008, 7:8 doi:10.1 186/1476-07I I-7-8

This article is available from: http://www.ann-clinmicrob.com/content/7/I/8

(c) 2008 Neonakis et al; licensee BioMed Central Ltd.

This is an Open Access article distributed under the terms of the Creative Commons Attribution License (http://creativecommons.org/licenses/by/2.0), which permits unrestricted use, distribution, and reproduction in any medium, provided the original work is properly cited.
Received: 14 October 2007

Accepted: 13 March 2008

\begin{abstract}
Background: Although tuberculosis is not uncommon among patients with myelodysplastic syndrome (MDS), only a few reports of such patients suffering from miliary tuberculosis (MT) exist. MT often presents as a fever of unknown origin and it is a curable disease, yet fatal if left untreated.

Case presentation: We report a case of MT with no clinical or laboratory indications of pulmonary involvement in a patient with MDS, and review the relevant literature. Mycobacterium tuberculosis was isolated from the liquid culture of a bone marrow aspirate.

Conclusion: Even if the initial diagnostic investigation for a fever of obscure etiology is negative, MT should not be excluded from the differential diagnosis list. Since it is a curable disease, persistent and vigorous diagnostic efforts are warranted. In suspected cases, mycobacterial blood cultures should be collected as soon as possible after hospital admission and early bone marrow aspirate with mycobacterial cultures is advocated.
\end{abstract}

\section{Introduction}

The increase in numbers and survival of immunocompromised individuals has contributed to the re-emergence of tuberculosis (TB) as a major public health problem. TB is common among patients with myelodysplastic syndrome (MDS) but only a few reports of such patients suffering from miliary tuberculosis (MT) exist. MT often presents as a fever of unknown origin (FUO). It is a curable disease, yet fatal if left untreated and therefore prompt diagnosis is mandatory.

\section{Case presentation}

A 64-year-old male with refractory anemia with multilineage dysplasia and ringed sideroblasts, was admitted with low grade fever, fatigue and insomnia of three months duration. He was initially diagnosed with MDS five years ago, having a single cytogenetic abnormality of trisomy 19. He was treated with recombinant human erythropoietin and pyridoxine. One year prior to admission he underwent a splenectomy for hypersplenism. Afterwards his hematological parameters improved and he was only 
occasionally transfused with packed red cells. On admission physical examination revealed low-grade fever $\left(37.5^{\circ} \mathrm{C}\right)$ and pallor of the skin. He had normochromic normocytic anemia (hemoglobin $9.8 \mathrm{~g} / \mathrm{dL}$ ), thrombocytopenia $(79,000 / \mu \mathrm{L})$ and a normal white blood count $(9,800 / \mu \mathrm{L})$ with dysplastic neutrophils and mild lymphopenia $(1,400 / \mu \mathrm{L})$. Biochemistry was normal, ESR was 44 $\mathrm{mm} / \mathrm{h}$ and CRP was $0.75 \mathrm{mg} / \mathrm{dl}$. Chest radiography, urine and blood cultures for common bacteria were negative. A bone marrow aspirate was performed, which revealed a hypercellular bone marrow with multilineage dysplasia, $24 \%$ ringed sideroblasts, $2 \%$ blasts and no additional cytogenetic abnormalities. Bone marrow trephine biopsy showed areas of hypercellularity without the presence of epithelioid cell granulomas or areas of necrosis. The aspirate was further cultured for mycobacteria. It was inoculated into MB/Bact Blood Culture Bottles for BacT/Alert 3D (bioMerieux, Durham, NC, USA) and LowensteinJensen (LJ) slants (bioMerieux, Marcy l' Etoile, France) and incubated at $37^{\circ} \mathrm{C}$. The acid-fast staining of the specimen was positive. The liquid culture turned positive eight days later. The isolate was identified as a member of the M. tuberculosis complex with gene probes [AccuProbe (GenProbe, San-Diego, USA)] and further differentiated as M. tuberculosis with biochemical analysis. Mycobacterial sputum cultures were negative. The chest computed tomography (CT) scan was normal. Drug susceptibilities were determined with the proportions method. The isolate was susceptible to all anti-tuberculosis drugs tested. The patient received a 4-drug regimen (isoniazid, rifampicin, ethambutol and pyrazinamide) for six months, followed by isoniazid and rifampicin for another three months. He defervesced shortly after the onset of treatment.

\section{Discussion}

The term "Miliary Tuberculosis" refers to all types of progressive disseminated hematogenous TB regardless of the pathological picture [1]. Its incidence is estimated at approximately $1 \%$ of all TB cases [2] and it is usually diagnosed when miliary infiltrates are found either on chest roentgenograms and CT scans or when there are autopsy or biopsy findings of miliary organ involvement [1]. However, in some cases such findings may not be evident in the radiological studies and the term "Disseminated Tuberculosis" has been proposed for such, whereas the term "Miliary Tuberculosis" is mostly reserved for cases of disseminated TB with miliary shadows [3].

MDS is a group of clonal myeloid disorders that often progress to acute leukemia [4]. Pancytopenia is a common finding. Cell-mediated immunity is seriously impaired and patients are predisposed to infections, which account for about one third of deaths. In a 10-year review from a comprehensive cancer center, the MDS cases with TB rep- resented $10.5 \%$ of the total number of patients diagnosed with TB and hematological malignancies [5]. MT was reported in 3\% of the cases of TB with malignancy. However, there was no report on whether MDS patients were included in this percentage. In a recent report regarding patients with hematological malignancies and TB, 7.2\% of them suffered from MDS, but none of the TB cases was reported as miliary [6]. The authors commented that MT was considered as rare in the population studied. In another study, the frequency of TB among MDS patients was found to be $7.7 \%$ [7]. Among those cases only one was reported as miliary according to the radiological pattern, but with no extrapulmonary involvement. Despite this relatively high frequency of TB among MDS patients, only two cases of extrapulmonary tuberculous infections in this population have been reported. The first was a localized tuberculous lymphadenopathy [4], whereas the second was a case of MT in a patient later diagnosed with MDS [8].

MT is a serious illness demanding timely response. Fever is a constant symptom and may take the form of FUO [1]. Other symptoms include malaise, anorexia, weight loss, night sweats, cough, headaches or abdominal pain. In one third of patients, hepatomegaly or splenomegaly can be found [1]. Hematological abnormalities such as chronic disease anemia, leucopenia with lymphopenia, thrombocytopenia or, more rarely, pancytopenia, are frequently described $[1,8,9]$. It has been suggested that if MT coexists with pancytopenia, the latter may reflect an underlying hematological disease (often leukemia or a preleukemic disorder) [9]. Chest X-ray or CT images may or may not reveal miliary infiltrates, as such lesions need weeks to develop or may not develop at all [1]. In most cases granulomas can be histologically demonstrated in the tissues (lung, liver, lymph node or bone marrow) [10].

Isolation of the mycobacterium provides a definite diagnosis of MT. The use of liquid media cultures has allowed the isolation of M. tuberculosis and the determination of its susceptibilities within days. The yield of acid-fast smears is usually low in the investigation of FUO $[11,12]$. TB is considered to be one of the most common infectious causes of FUO, with high mortality rates $[1,9,12]$, so early diagnosis and treatment is important.

One question raised is whether a physician should perform an early bone marrow aspirate or whether blood cultures are sufficient for the isolation of mycobacteria in probable disseminated cases. In a recent study evaluating the diagnostic yield of blood and bone marrow cultures, it was suggested that disease caused by members of the $M$. tuberculosis complex could be misdiagnosed without a bone marrow culture [13]. The combined use of blood and bone marrow cultures can provide maximum sensi- 
tivity for the diagnosis of disseminated mycobacterial infections. If mycobacterial infection is suspected, blood cultures should be collected as soon as possible after hospital admission as they are usually negative when collected later [14].

It is important to emphasize that the forms of TB that most often cause FUO are either the disseminated form without the characteristic miliary pattern or extrapulmonary TB without clear localized features [10]. Physicians usually include TB in their differential diagnosis list when taking care of patients with a prolonged fever of obscure origin. However, in the absence of typical chest X-ray data, and as the initial specimen (usually sputum) acid-fast staining is negative and the clinical manifestations are not specific, TB as an etiology is usually by-passed and the diagnosis (if any) is delayed.

In conclusion, we have reported a rare case of MT with no clinical or laboratory indications of pulmonary involvement in a patient with MDS. Even if the initial diagnostic investigation for fever of obscure origin is negative, MT should not be excluded from the differential diagnosis list. As MT is a treatable disease, persistent and vigorous diagnostic efforts are warranted. In suspected cases, mycobacterial blood cultures should be collected as soon as possible after hospital admission and an early bone marrow aspirate with mycobacterial cultures is advocated.

\section{Conclusion}

- Even if the initial diagnostic investigation for fever of obscure etiology is negative, miliary tuberculosis (MT) should not be excluded from the differential diagnosis list.

- MT is a curable disease, thus persistent and vigorous diagnostic efforts are warranted.

- In suspected cases, mycobacterial blood cultures should be collected as soon as possible after hospital admission and early bone marrow aspirate with mycobacterial cultures should be performed.

\section{Abbreviations}

ESR: Erythrocyte Sedimentation Rate, CRP: C-Reactive Protein.

\section{Competing interests}

The author(s) declare that they have no competing interests.

\section{Authors' contributions}

IN carried out the laboratory procedures and drafted the paper. MA and GT handled the patient clinically. ZG participated in carrying out the laboratory procedures. EK revised the manuscript critically. DS conceived the report, and gave final approval of the submitted version. All authors read and approved the final manuscript.

\section{Acknowledgements}

Written consent was obtained from the patient.

\section{References}

I. Mert A, Bilir M, Tabak F, Ozaras R, Ozturk R, Senturk H, Aki H, Seyhan N, Karayel T, Aktuglu Y: Miliary tuberculosis: clinical manifestations, diagnosis and outcome in $\mathbf{3 8}$ adults. Respirology 200I, 6(3):217-224.

2. Rieder HL, Snider DE Jr, Cauthen GM: Extrapulmonary tuberculosis in the United States. Am Rev Respir Dis 1990, I 4 I (2):347-35I.

3. Matsushima T: Miliary tuberculosis or disseminated tuberculosis. Intern Med 2005, 44(7):687.

4. Al-Attia HM, Shanaa ZA, Knox-Macaulay HH: Localized tuberculous lymphadenopathy associated with the myelodysplastic syndrome. South Med J 1996, 89(3):319-320.

5. De La Rosa GR, Jacobson KL, Rolston KV, Raad II, Kontoyiannis DP, Safdar A: Mycobacterium tuberculosis at a comprehensive cancer centre: active disease in patients with underlying malignancy during 1990-2000. Clin Microbiol Infect 2004, I 0(8):749-752.

6. Silva FA, Matos JO, de QMFC, Nucci M: Risk factors for and attributable mortality from tuberculosis in patients with hematologic malignances. Haematologica 2005, 90(8): IIIO-III5

7. Kim HC, Goo JM, Kim HB, Lee JW, Seo JB, Im JG: Tuberculosis in patients with myelodysplastic syndromes. Clin Radiol 2002, 57(5):408-4|4.

8. Hunt BJ, Andrews V, Pettingale KW: The significance of pancytopenia in miliary tuberculosis. Postgrad Med J 1987, 63(743):801-804.

9. Maartens G, Willcox PA, Benatar SR: Miliary tuberculosis: rapid diagnosis, hematologic abnormalities, and outcome in 109 treated adults. Am J Med 1990, 89(3):29l-296.

10. Arnow PM, Flaherty JP: Fever of unknown origin. Lancet 1997, 350(9077):575-580.

II. Yuen KY, Woo PC: Tuberculosis in blood and marrow transplant recipients. Hematol Oncol 2002, 20(2):5 I-62.

12. Chin C, Lee SS, Chen YS, Wann SR, Lin HH, Lin WR, Huang CK, Kao $\mathrm{CH}$, Yen MY, Liu YC: Mycobacteriosis in patients with fever of unknown origin. J Microbiol Immunol Infect 2003, 36(4):248-253.

13. Pacios E, Alcala L, Ruiz-Serrano MJ, de Viedma DG, Rodriguez-Creixems M, Marin-Arriaza M, Berenguer J, Bouza E: Evaluation of bone marrow and blood cultures for the recovery of mycobacteria in the diagnosis of disseminated mycobacterial infections. Clin Microbiol Infect 2004, I 0(8):734-737.

14. Hanscheid T, Monteiro C, Cristino JM, Lito LM, Salgado MJ: Growth of Mycobacterium tuberculosis in conventional BacT/ ALERT FA blood culture bottles allows reliable diagnosis of Mycobacteremia. J Clin Microbiol 2005, 43(2):890-89|

Publish with Bio Med Central and every scientist can read your work free of charge

"BioMed Central will be the most significant development for disseminating the results of biomedical research in our lifetime. "

Sir Paul Nurse, Cancer Research UK

Your research papers will be:

- available free of charge to the entire biomedical community

- peer reviewed and published immediately upon acceptance

- cited in PubMed and archived on PubMed Central

- yours - you keep the copyright 